\title{
Proyecciones de población para áreas pequeñas: estimaciones a nivel de parroquia en cinco provincias del noroeste de España
}

\author{
Esteban Fernández Vázquez ${ }^{* a}$ / Fernando Rubiera Morollón ${ }^{b}$ \\ aUniversidad de Oviedo - Laboratorio de Análisis Económico Regional (REGIOlab) \\ bUniversidad de Oviedo - Departamento de Economía Aplicada
}

Recibido: 21 de septiembre de 2020 / Aceptado: 5 de octubre de 2020

\begin{abstract}
Resumen
En este artículo se presenta un procedimiento sencillo para obtener proyecciones demográficas para áreas geográficas pequeñas. El método aquí presentado se basa en asumir que las tendencias de las zonas que se van a proyectar serán similares a las observadas en períodos anteriores, y en tomar las proyecciones para áreas geográficas más extensas como punto de referencia. En términos matemáticos, esta técnica pasa por un problema de minimización restringida de las divergencias cuadráticas entre las proyecciones obtenidas y las observadas en períodos pasados, donde las proyecciones demográficas agregadas se toman como restricción. Esta propuesta encaja adecuadamente con el escenario presente en el caso de las proyecciones demográficas que el Instituto Nacional de Estadística (INE) elabora para España, donde las previsiones se presentan a escala provincial pero no con más nivel de detalle. Aunque estas proyecciones provinciales son útiles de cara a la planificación de políticas y a la dotación de determinados servicios por parte del sector público, sería deseable disponer de ellas a una escala geográfica inferior al ámbito provincial. El procedimiento presentado en este trabajo se ilustra con el caso de cinco provincias de España, correspondientes a las comunidades autónomas de Asturias y Galicia, en las que las predicciones de población correspondientes para el año 2033 son desagregadas a una escala geográfica de parroquia.
\end{abstract}

Palabras clave

Proyecciones demográficas / Información desagregada / Parroquias de Asturias y Galicia.

\section{Population forecast for small areas: estimates for the parishes of five northwestern Spanish provinces}

\begin{abstract}
This article presents a simple procedure for obtaining demographic projections for small geographic areas. The method presented here is based on assuming that the trends of the areas to be projected will be similar to those observed in previous periods and taking the projections for larger geographic areas as a reference point. In mathematical terms, this technique involves a problem of restricted minimization of the quadratic divergences between the projections obtained and those observed in past periods, where the aggregate demographic projections are taken as a constraint. This proposal fits very well with the present scenario in the case of the demographic projections that the National Institute of Statistics prepares for Spain, where these forecasts are presented at a provincial level but not in any more detail. While these provincial projections are useful for policy planning and the provision of certain services by the public sector, it would be desirable to have them at a more detailed geographical scale at the provincial level. The procedure presented in this paper is illustrated with the case of five provinces in Spain, corresponding to the Autonomous Communities of Asturias and Galicia, in which the population forecasts for those provinces for the year 2033 are broken down to a parish geographical scale.
\end{abstract}

\section{Keywords}

Demographic projections / Disaggregated data / Asturian and Galician parishes.

JEL Codes: J11, C53, R23.

\footnotetext{
*Correspondencia autor: evazquez@uniovi.es
} 


\section{Introducción}

Estamos asistiendo a un proceso de concentración de la población global sin precedentes en la historia de la humanidad. En la actualidad, más del 55\% de la población mundial vive en ciudades, y según las estimaciones de Naciones Unidas (ONU, 2019), para el año 2050 el porcentaje excederá el 65\%. En palabras de Glaeser, Kourtit y Nijkamp (2020), el siglo XXI es el siglo de los "imperios urbanos: un nuevo orden económico mundial dominado por las ciudades, donde la población se ha concentrado radicalmente". Esta dinámica global de concentración poblacional se reproduce en la escala nacional, regional e incluso intrarregional. Según Naciones Unidas (ONU, 2019), el 73\% de la población europea vive en áreas urbanas, y este porcentaje se eleva al 79\% en el caso de España, donde observamos como se produce simultáneamente un imparable crecimiento de las principales ciudades del país, y sus entornos. junto con una acelerada despoblación de amplias áreas.

La investigación sobre la evolución de la distribución espacial de la población ha centrado su atención, preferentemente, en la comprensión los patrones globales que están detrás de estos procesos de concentración extrema de la población. Se ha buscado identificar los factores que impulsan el crecimiento acelerado de las áreas urbanas, o la generación de procesos centro/periferia, prestando, por el contrario, muy poca atención a las dinámicas poblacionales intrarregionales o locales. Sin embargo, como señalan Glaeser, Ponzetto y Tobio (2014), la verdadera comprensión de los procesos espaciales de localización de la población y la articulación de políticas verdaderamente efectivas requiere entender los comportamientos intrarregionales donde se esconden marcadas heterogeneidades.

En muchas ocasiones, la ausencia de estos estudios regionales/locales está motivada por la falta de una adecuada información o bien por la ausencia de proyecciones fiables de población a escala local. En el caso de España, el INE elabora proyecciones de población que desagrega espacialmente por provincias. No se puede disponer, por tanto, de proyecciones demográficas por debajo del nivel provincial, con lo que no se puede analizar la tendencia de las ciudades medianas o pequeñas, o anticipar la dinámica previsible de las zonas rurales.

El objetivo de este trabajo es ofrecer un procedimiento que nos permita disponer de proyecciones desagregadas en el ámbito local que sean coherentes con las que realiza el INE a escala provincial. Esta información posibilitaría entender y anticipar los cambios en el modelo de asentamiento de la población en España, pudiendo comparar la previsible evolución de las grandes ciudades con las medianas o pequeñas, o pudiendo comprender las dinámicas de las zonas rurales dependiendo de su localización en el mapa nacional. A modo de ilustración de la capacidad de este procedimiento, se ha aplicado para desagregar las proyecciones de población del INE en los casos de Asturias y Galicia, dos regiones del noroeste peninsular especialmente afectadas por el declive demográfico y que parecen estar experimentando cambios en su modelo de distribución territorial de la población. La aplicación se ha realizado empleando un amplio nivel de desagregación espacial, el ámbito de la parroquia, lo que nos proporciona una perspectiva muy detallada y espacialmente precisa del previsible comportamiento de la población en los próximos años.

El trabajo se estructura como sigue. En el apartado siguiente se resume el procedimiento de elaboración de proyecciones del INE, mostrando sus resultados para las provincias en las que aplicaremos nuestro análisis. En el apartado tercero se presenta la propuesta metodológica de este trabajo. En el apartado cuarto se resumen las variables que se han usado para aplicar esta propuesta al caso de Galicia y Asturias. Los resultados, con un breve comentario, se muestran en el apartado quinto. Se completa el trabajo con un apartado final de conclusiones.

\section{Proyecciones demográficas a nivel provincial: metodología oficial aplicada por el INE y cifras para las provincias analizadas}

Las proyecciones demográficas son un instrumento de extraordinaria relevancia en la toma de decisiones, diseño de políticas socioeconómicas, programación de infraestructuras u ordenación del te- 
rritorio. Se han desarrollado múltiples metodologías que sofistican los procedimientos y hacen más precisas y fiables las predicciones ${ }^{1}$. En el caso español, el INE elabora anualmente La proyección de la población de España. La metodología utilizada por el INE es la mas estándar internacionalmente, basada en el método clásico de componentes. La aplicación de dicho método responde al siguiente esquema: partiendo de la población residente en un cierto ámbito geográfico y de los datos observados para cada uno de los componentes demográficos básicos -mortalidad, fecundidad y migración-, se trata de deducir cuál sería la población correspondiente en fechas posteriores bajo ciertas hipótesis sobre el devenir de esos tres fenómenos, que son los que determinan su crecimiento y la estructura por edades.

Con la información demográfica más actualizada disponible, se analiza retrospectivamente cada uno de los componentes demográficos básicos y se establecen hipótesis sobre su incidencia futura en cada nivel territorial considerado en cada año del período que se va a proyectar. Concretamente, se establecen hipótesis sobre las tasas específicas de fecundidad por generación, las tasas específicas de mortalidad por sexo y generación, las tasas específicas por sexo y generación de emigración exterior y de migración interior interprovincial, así como en flujos de inmigración exterior para cada sexo y generación.

Las proyecciones de población residente en España a 1 de enero de cada año del período proyectado para cada una de sus provincias se obtienen de acuerdo con un modelo de proyección multiregional. Así, se proporcionan datos sobre población en cada uno de los niveles territoriales considerados, y también las cifras proyectadas de nacimientos, defunciones y movimientos migratorios que tendrán lugar en cada uno de los años del período considerado. Todas las estimaciones guardan la necesaria coherencia entre flujos y stocks demográficos, y la debida consistencia interterritorial. Partiendo de la población residente en cada nivel territorial considerado de sexo $s$ y edad $x$ a fecha 1 de enero del año $\left(P_{s, x}^{t}\right)$, se obtiene la proyección de población residente de edad $x+1$ y sexo $s$ en dicha área geográfica a 1 de enero del año $t+1\left(P_{s, x+1}^{t+1}\right)$, a partir de la siguiente fórmula:

$$
P_{s, x+1}^{t+1}=\frac{\left[1-0,5\left(m_{s, x}^{t}+e_{s, x}^{t}\right)\right] P_{s, x}^{t}+I M_{S, x}^{t}}{\left[1+0,5\left(m_{s, x}^{t}+e_{s, x}^{t}\right)\right]}
$$

donde $m_{s, x}^{t}$ es la tasa de mortalidad en el año $t$ de la generación de individuos residentes en España de sexo $s$ y edad $x$ a 1 de enero del año $t ; e_{s, x}^{t}$ es la tasa de emigración exterior en el año $t$ de la generación de individuos residentes en España de sexo $s$ y edad $x$ a 1 de enero del año $t ; I M_{s, x}^{t}$ es el flujo de inmigración procedente del extranjero en el año $t$ de individuos de sexo $s$ y edad $x$ a 1 de enero del año $t$.

Para implementar este modelo de predicción multirregional, se resuelve un sistema de 52 ecuaciones, una por provincia, en el que se incorporan los datos de movimientos migratorios intrarregionales y hacia el/desde el extranjero. Un aspecto que incrementa la precisión y sofisticación de las predicciones es la modelización de la evolución de la fecundidad teniendo en cuenta el comportamiento marcadamente diferenciado entre las mujeres españolas y extrajeras, así como la cifra de mujeres en edad fecunda a lo largo del tiempo. Para incorporar estas consideraciones, en primer lugar se modeliza la serie retrospectiva de tasas específicas de fecundidad por edad y nacionalidad, asumiendo una evolución logarítmica lineal en el tiempo que diferencia mujeres españolas y extrajeras. Esta relación se estima mediante mínimos cuadrados ordinarios (MCO), y las estimaciones obtenidas se aplican por provincias a partir de una simulación del comportamiento diferencial de la fecundidad en cada provincia respecto del total nacional, así como la evolución de la edad mediana de maternidad y del rango intercuartílico de las tasas de fecundidad de cada una de ellas. A partir de dichos parámetros, se derivan unas nuevas proyecciones de tasas de fecundidad provincial siguiendo un modelo Brass-Gompertz relacional, que es un modelo estándar en la literatura especializada.

Ciñéndolos a las provincias en las que se centrará este trabajo, las proyecciones del INE se muestran en la Tabla 1, en la que, además de mostrar la variación proyectada por el INE para las provincias

${ }^{1}$ Para una revisión puede verse, entre otros, Mazzuco y Kelman (1990). 
de Asturias y Galicia en el período 2019-2033, también se efectúa una comparativa con el período 2005-2019.

Estas cifras de población, junto con las obtenidas para el año 2033, permiten calcular densidades de población, que para los años 2005, 2019 y 2033 se recogen en la Tabla 2.

Tabla 1. Variación de la población en el período 2005-2019 y proyección estimada para 2019-2033

\begin{tabular}{lcc}
\hline & $\begin{array}{c}\text { Tasa de crecimiento } \\
2005-2019(\%)\end{array}$ & $\begin{array}{c}\text { Tasa de crecimiento estimada } \\
2019-2033(\%)\end{array}$ \\
\hline Asturias & $-3,82$ & $-8,02$ \\
A Coruña & 1,09 & $-4,28$ \\
Lugo & $-6,73$ & $-7,69$ \\
Ourense & $-8,12$ & $-8,57$ \\
Pontevedra & 2,03 & $-3,77$ \\
\hline
\end{tabular}

Fuente: elaboración propia a partir de los datos del INE, Cifras de población y proyecciones demográficas.

Tabla 2. Densidad de población en los años 2005 y 2019 y proyección de la densidad para 2033

\begin{tabular}{lccc}
\hline & \multicolumn{3}{c}{ Habitantes por kilómetro cuadrado } \\
\cline { 2 - 4 } & 2005 & 2019 & Proyección 2033 \\
\hline Asturias & 100,23 & 96,41 & 88,68 \\
A Coruña & 139,62 & 141,13 & 135,09 \\
Lugo & 35,85 & 33,44 & 30,87 \\
Ourense & 46,07 & 42,33 & 38,70 \\
Pontevedra & 205,23 & 209,39 & 201,51 \\
\hline
\end{tabular}

Fuente: elaboración propia a partir de los datos del INE, Cifras de población y proyecciones demográficas.

\section{Metodología para la realización de desagregaciones espaciales de las proyecciones demográficas}

La máxima desagregación espacial en la que se presentan las proyecciones demográficas del INE es el nivel provincial. Esto limita seriamente su posible uso en decisiones o diseño de políticas de ámbito territorial más detallado (áreas intraprovinciales o entornos urbanos locales). Nuestro objetivo es disponer de proyecciones con un mayor detalle de desagregación espacial. No buscamos realizar nuevas proyecciones, sino desarrollar un método de desagregación de las existentes. Esto simplifica la aproximación metodológica, dado que no necesariamente precisamos estimar proyecciones con base en la información desagregada disponible. Podemos confiar en la calidad de las proyecciones agregadas y sobre ellas aplicar un procedimiento de desagregación. Existen múltiples metodologías con este enfoque $^{2}$.

Nuestra propuesta en este trabajo se basa en el uso de técnicas shift-share (Dunn, 1960), específicamente en el método descrito en Alonso, Fernández Vázquez y Rubiera (2015). Este enfoque destaca por su simplicidad, y ya ha ido empleado con fines predictivos de series de empleo ${ }^{3}$. Por el modo en el

2 Véase King, Rosen y Tanner (2014) para una emplia revisión de distintos enfoques y procedimientos.

${ }^{3}$ En Mayor, López y Pérez (2017) se presentan las ventajas e inconvenientes del procedimiento y una evaluación de su fiabilidad. 
que se construye el procedimiento, permite disponer de desagregaciones locales consistentes con la información agregada, lo que resulta especialmente interesante, en nuestro caso, para que las proyecciones locales sean coherentes con la información oficial a escalas superiores.

Así, proponemos aplicar este procedimiento a las proyecciones del INE a escala provincial para desagregarlas en el ámbito parroquial. La aplicación se basa en dos supuestos fundamentales:

i. Las variaciones de población estimadas a escala de cada parroquia $i$ entre los años 2019 y $2033\left(\tilde{g}_{i}\right)$ se espera que sean similares a las registradas a esa misma escala en el período 2005-2019 $\left(g_{i}\right)$.

ii. Las proyecciones provinciales para el año $2033(\tilde{p})$ se toman como ciertas.

Así, la técnica de estimación se formula como un proceso de optimización restringida donde (i) permite definir la función objetivo mientras que (ii) fija las restricciones que se deben cumplir por las soluciones obtenidas en cada provincia.

En concreto, se expone un problema de optimización restringida como:

$$
\operatorname{Min} \sum_{i=1}^{M}\left[\tilde{g}_{i}-g_{i}\right]^{2} p_{i}^{0}
$$

sujeto a:

$$
\sum_{i=1}^{M} \tilde{g}_{i} p_{i}^{0}=\tilde{p}
$$

La ecuación (2) establece el criterio de distancia en términos cuadráticos entre las diferenciales previstas y las observadas, ponderadas por el tamaño de la población en la parroquia considerada en el período inicial $2019\left(p_{i}^{0}\right)$. Esta expresión se minimiza sujeta a la restricción presente en la ecuación (3), que garantiza que las tasas proyectadas en el ámbito de la parroquia son consistentes con las previstas por el INE para el total de la provincia $(\tilde{p})$. Una vez resuelto este programa de optimización, se obtienen las tasas de variación de población $\tilde{g}_{i}$ para cada parroquia $i$, considerando como horizonte de predicción el año 2033.

\section{Aplicación a las variaciones de población entre 2019 y 2033 de las parroquias en Asturias y Galicia}

A modo de ilustración, proponemos aplicar la metodología propuesta a las parroquias que el nomenclátor de Asturias y Galicia permite identificar. La parroquia es una entidad de ámbito territorial inferior al municipio. Algunas comunidades, entre las que se encuentran Asturias y Galicia, les han otorgado carácter administrativo con el fin de coordinar y organizar la actividad comunitaria en dicho entorno. El nomenclátor de Asturias distingue un total de 856 parroquias, mientras que en el caso de Galicia podemos identificar 3.787. Asturias tiene 78 municipios y Galicia 313. Estamos trabajando, por tanto, con un nivel de desagregación más de diez veces superior al municipal, lo que nos permitirá disponer de una proyección extraordinariamente precisa desde la perspectiva espacial.

Sobre estas unidades espaciales se ha aplicado el problema de estimación recogido en las ecuaciones (2) y (3) y se ha resuelto para cada una de las cinco provincias estudiadas en este trabajo. Las tablas y figuras presentadas en esta sección y en los Apéndices 1 y 2 resumen los resultados obtenidos.

A modo de resumen general, la Tabla 3 establece una comparación de la variación de la población entre los años 2005 y 2019 con la del período estimado 2019-2033. Para sintetizar, se muestran solo los valores máximo y mínimo por provincia de entre las parroquias de mas de 2.000 habitantes en el año 2019. A través de estos valores máximos y mínimos se puede constatar la gran heterogeneidad existente en el ámbito espacial estudiado. Hay parroquias que experimentan crecimientos superiores al $100 \%$, mientras que otras decrecen a ritmos superiores al $-20 \%$. 
Tabla 3. Variación de la población en el período 2005-2019 y proyección estimada para 2019-2033. Valores máximo y mínimo por provincia (parroquias mayores de 2.000 habitantes en el año 2019)

\begin{tabular}{|c|c|c|c|c|}
\hline Provincia & Municipio & Parroquia & $\begin{array}{c}\text { Tasa de } \\
\text { crecimiento } \\
\text { 2005-2019 } \\
(\%)\end{array}$ & $\begin{array}{c}\text { Tasa de } \\
\text { crecimiento } \\
\text { estimada } \\
2019-2033 \\
(\%)\end{array}$ \\
\hline \multirow{2}{*}{ Asturias } & Valdés & Luarca & $-49,98$ & $-29,54$ \\
\hline & Avilés & San Cristóbal de Entreviñas & 82,84 & 124,67 \\
\hline \multirow{2}{*}{ A Coruña } & Cariño & Cariño (San Bartolomeu) & $-19,78$ & $-20,63$ \\
\hline & A Coruña & San Cristovo das Viñas & 134,43 & 96,46 \\
\hline \multirow{2}{*}{ Lugo } & Cervo & Lieiro (Santa María) & $-10,95$ & $-14,57$ \\
\hline & Ribadeo & Ribadeo (Santa María) & 18,99 & 15,37 \\
\hline \multirow{2}{*}{ Ourense } & A Rúa & A Rúa de Valdeorras (Santo Estevo) & $-13,43$ & $-17,07$ \\
\hline & Barbadás & A Valenzá (San Bernabeu) & 59,55 & 55,91 \\
\hline \multirow{2}{*}{ Pontevedra } & Ponte Caldelas & Ponte Caldelas (Santa Eulalia) & $-12,18$ & $-19,92$ \\
\hline & Vigo & Navia (San Paio) & 178,25 & 170,51 \\
\hline
\end{tabular}

Fuente: elaboración propia a partir de los datos del INE, Cifras de población y proyecciones demográficas.

Para reforzar esta visión de conjunto, en la Figura 1 se muestra una comparativa de la densidad de población existente en Asturias y Galicia en el año 2019 y la proyectada para 2033. Tanto Asturias como Galia presentan una significativa reducción de densidad de población en el período proyectado. Se observa que ese período (2019-2033) sigue la senda de las décadas precedentes con comportamientos muy similares, aunque con una significativa caída de la densidad media en ambos casos.

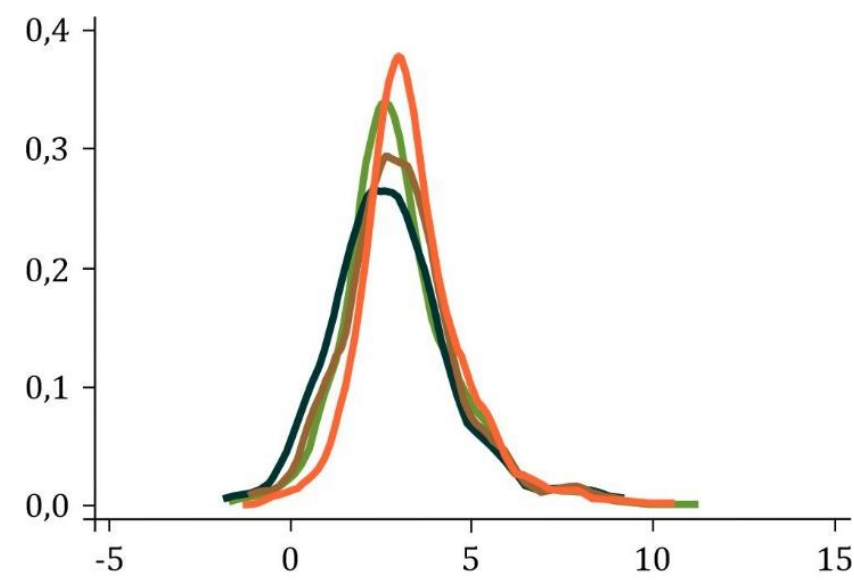

Densidad de población. Hab./ $\mathrm{km}^{2}$ (en logaritmos)

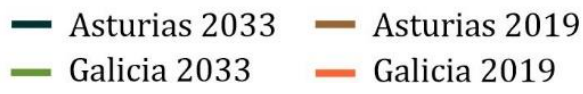

Figura 1. Densidades de población en Asturias y Galicia, comparativa entre 2019 y 2033. Fuente: elaboración propia. 
En la Figura 2 se relaciona la tasa de crecimiento de las parroquias en el período estimado (eje de ordenadas) y la densidad de población en el año 2019 (eje de abscisas). La nube de puntos que se obtiene muestra la heterogeneidad existente a la que antes hacíamos referencia. Hay parroquias que, partiendo de densidades bajas, experimentan un gran crecimiento, junto con otras que tienen un comportamiento totalmente opuesto. Esta Figura 2 se puede interpretar como un análisis de convergencia: si la recta de estimación de la nube de puntos fuera negativa y significativa, indicaría una tendencia a converger en densidades de población; por el contrario, una nube de puntos positiva y significativa estaría indicando una tendencia a la divergencia. Lo que observamos que la nube de puntos tiene tendencia positiv,a de lo que se deduce que las desigualdades en densidad tenderán a incrementarse en el período proyectado.

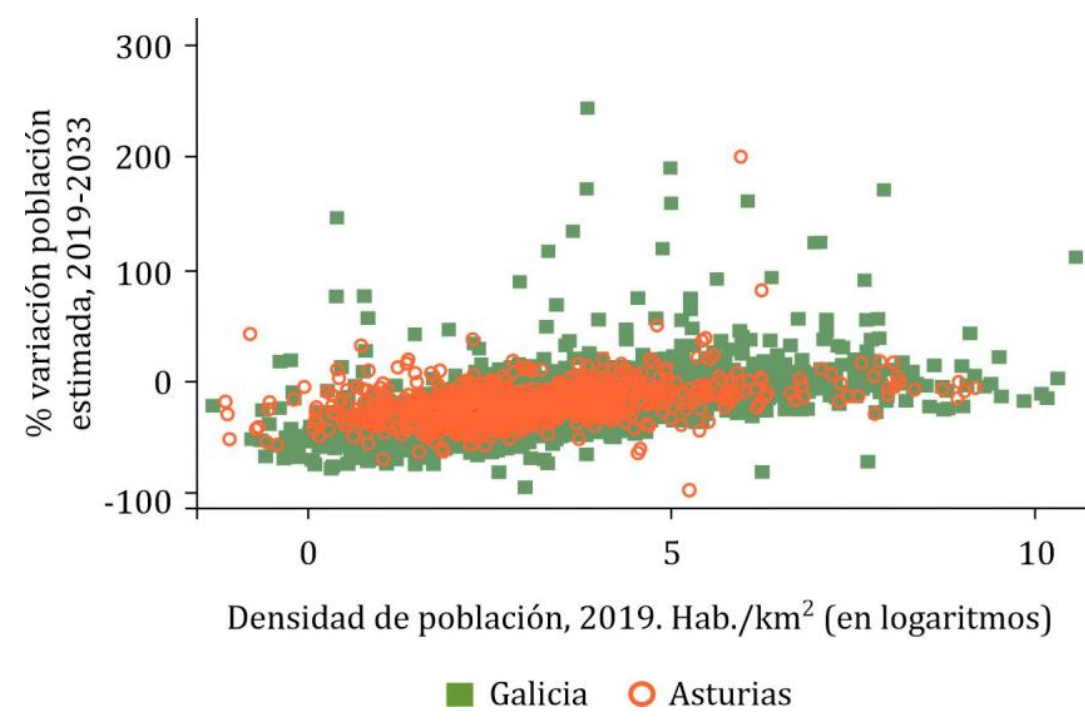

Figura 2. Densidades de población en 2019 y crecimiento estimado 2019-2033. Fuente: elaboración propia.

Para llegar a un análisis más detallado que permita aprovechar la información generada con la proyección desagregada realizada, se necesita observar el comportamiento territorial de la densidad de población de modo más preciso. Esto es lo que se realiza en los mapas siguientes, donde se presenta primero la densidad de población estimada por parroquias para Asturias (Figura 3) y para Galicia (Figura 4), y después la variación porcentual estimada de población por parroquias en Asturias (Figura 6) y en Galicia (Figura 7). Para el caso de Galicia se incluyen en el Apéndice 1 los mismos mapas por provincias. Adicionalmente, en el Apéndice 2 se presentan dos mapas en los que se recogen tanto para Asturias como para Galicia las variaciones porcentuales de población para el período 2005-2019 con fines puramente comparativos.

Todos estos mapas ilustran con mayor claridad lo que se apuntaba a partir de las Figuras 1 y 2 . Las desigualdades espaciales en la distribución de la población se agravarán significativamente en los próximos años. En el caso de Asturias puede verse como hay una clara concentración en torno a las parroquias más dinámicas del área central y algunas parroquias de la costa, especialmente la oriental. Casi todas las parroquias rurales pierden población, con la excepción de algunos de los principales núcleos de población del entorno rural. El comportamiento de Galicia es muy similar, aunque con algunos matices. Igual que en Asturias, las parroquias más dinámicas de las áreas urbanas de A Coruña, Santiago, Vigo, Pontevedra y Lugo son las más capaces para incrementar su densidad de población. Cuando nos alejamos de estos núcleos urbanos, la tónica general es experimentar variaciones negativas de densidad de población. El efecto de la costa no es tan marcado como en Asturias: solo en la costa atlántica-sur, en el entorno de Vigo, y en la costa cantábrica-norte, alrededor de A Coruña, se proyec- 
tan crecimientos de densidad significativos. En el interior, la pérdida de población es general, con la excepción, igual que ocurría en Asturias, de algunas cabeceras rurales.

Los patrones proyectados son similares con los que se muestran en otros trabajos previos, como Rubiera, Fernández, Gutiérrez y Viñuela (2014), y coherentes con modelos de localización poblacional como el desarrollado por Gutiérrez, Rubiera y Viñuela (2017), que analizan la existencia de un cambio de modelo de asentamiento poblacional en el noroeste peninsular. Frente al modelo de ocupación general del territorio que dominó el siglo XX, donde la densidad de población descendía lentamente según nos alejábamos de los principales entornos urbanos, en el siglo XXI avanzamos rápidamente a un modelo de fuerte concentración de la población tanto a escala provincial, alrededor de las principales ciudades, como a escala local, alrededor de las villas o núcleos rurales más poblados. La densidad de población, ahora, cae drásticamente en cuanto nos alejamos de estos núcleos principales, con una tendencia a dejar despoblado la mayor parte del territorio. La población tiende a concentrarse en focos muy concretos, y tiende a desaparecer la aldea tradicional, entendida como núcleos de muy poca población pero dispersos a lo largo del territorio. Por ello, se observa una recuperación y una aproximación a las ciudades de las especies animales autóctonas, que están ocupando el espacio que ha dejado el hombre en las zonas rurales.

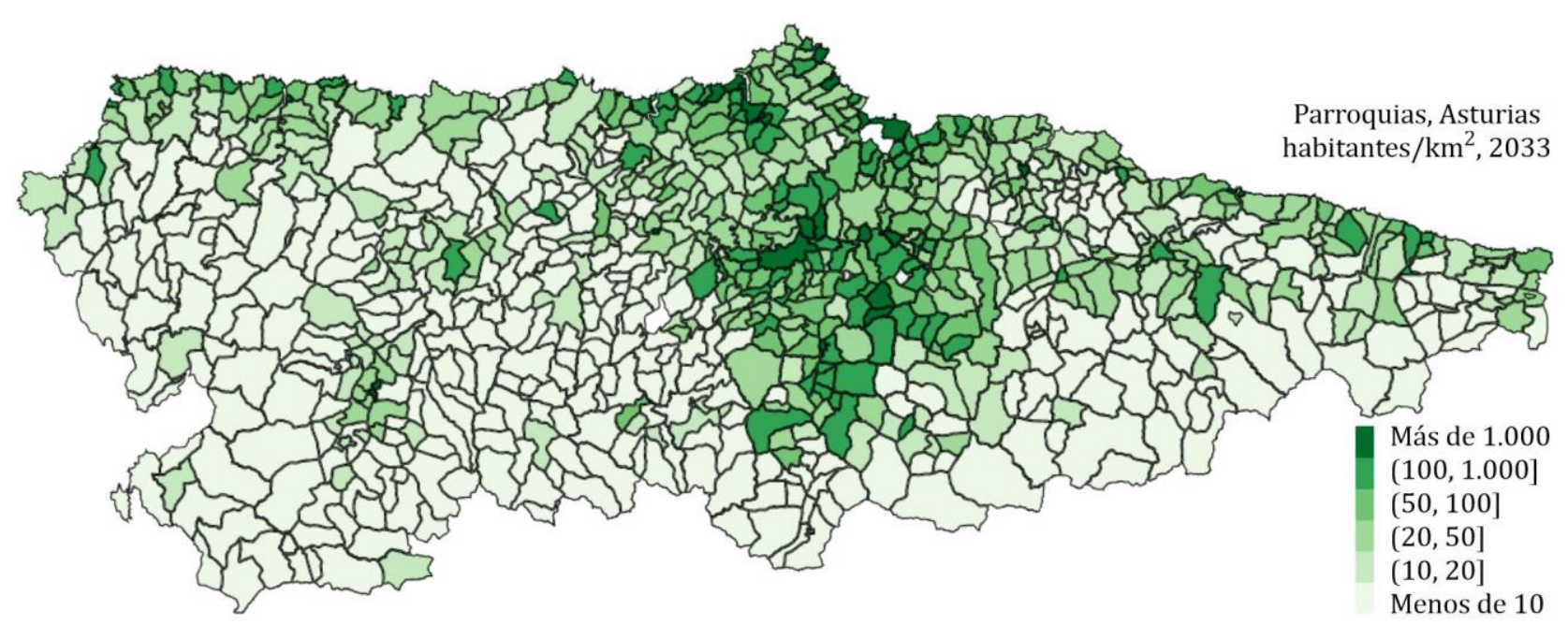

Figura 3. Densidad de población estimada de las parroquias asturianas, 2033. Fuente: elaboración propia.

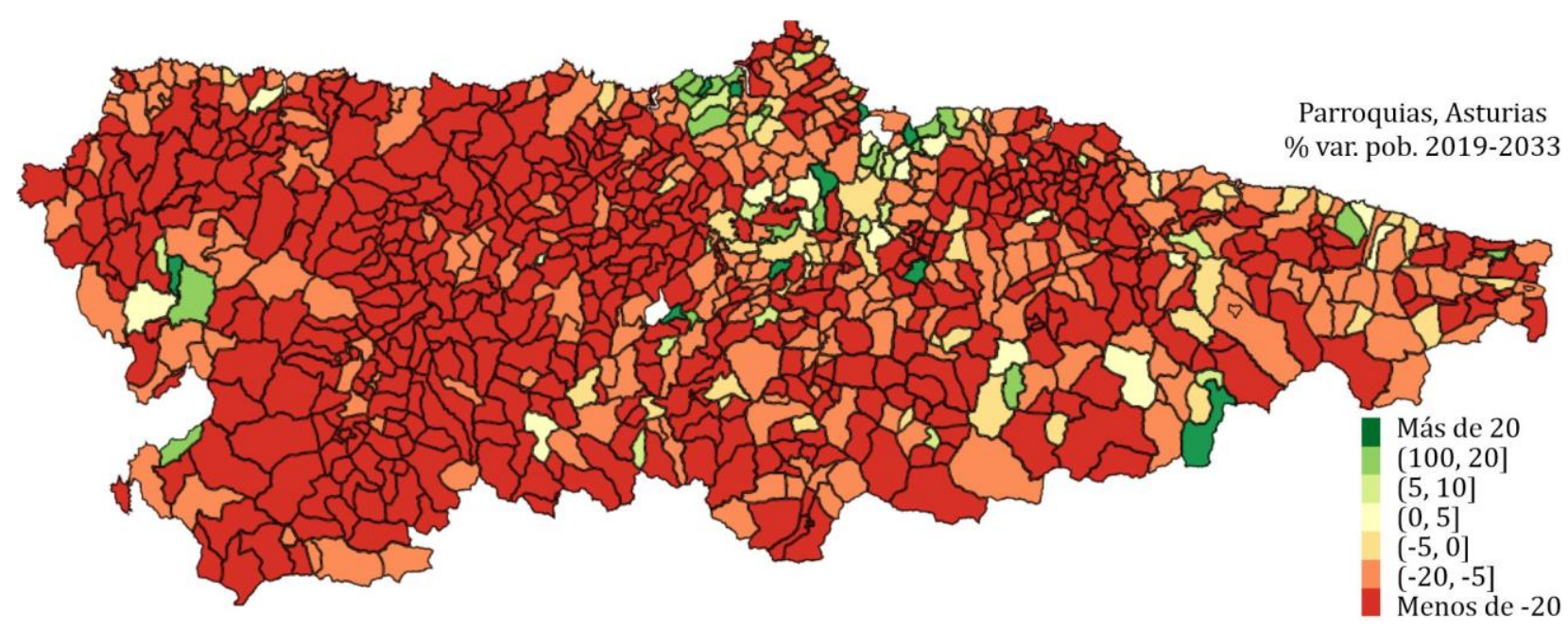

Figura 4. Variación porcentual estimada de la población parroquias asturianas, 2019-2033. Fuente: elaboración propia. 


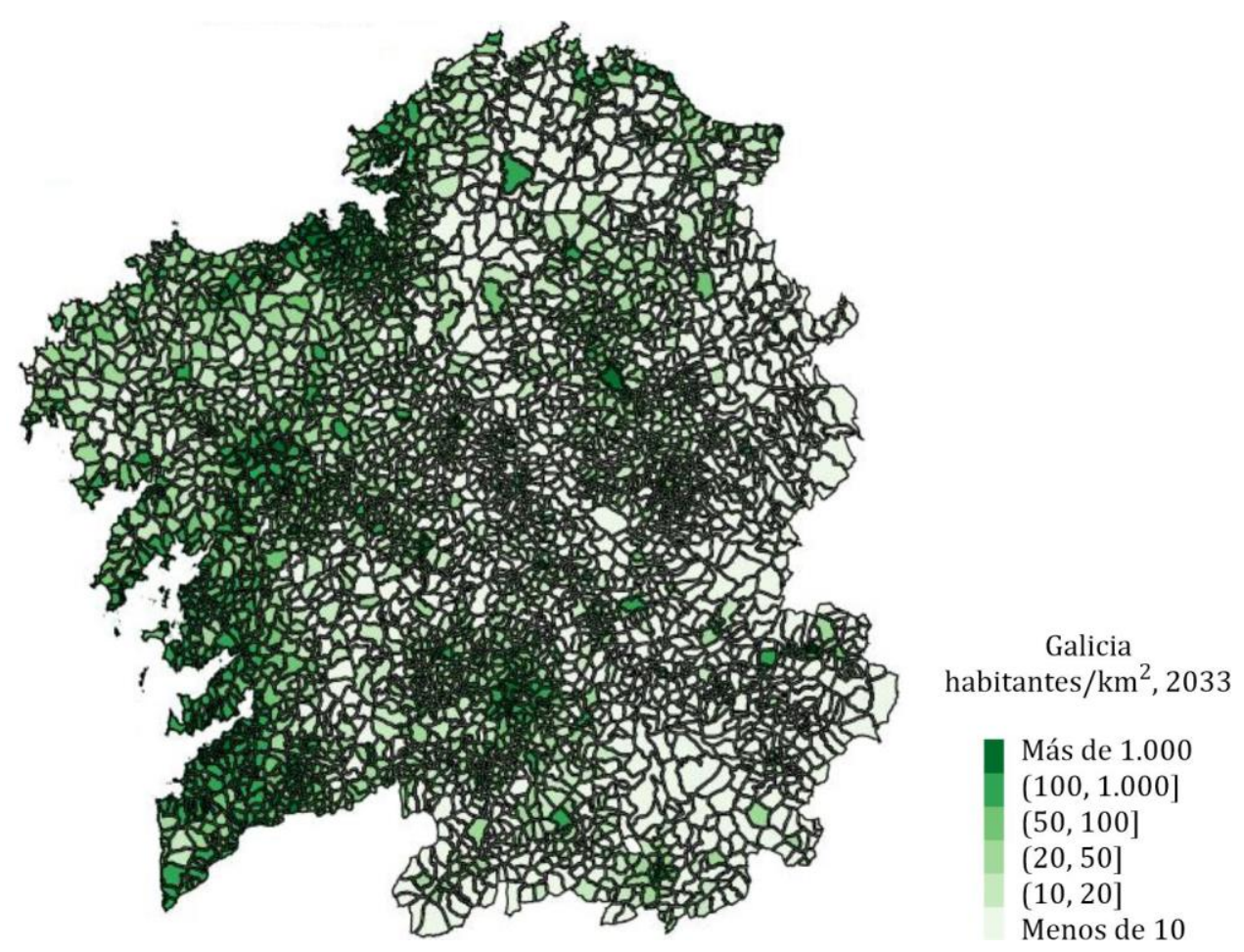

Figura 5. Densidad de población estimada de las parroquias gallegas, 2033. Fuente: elaboración propia.

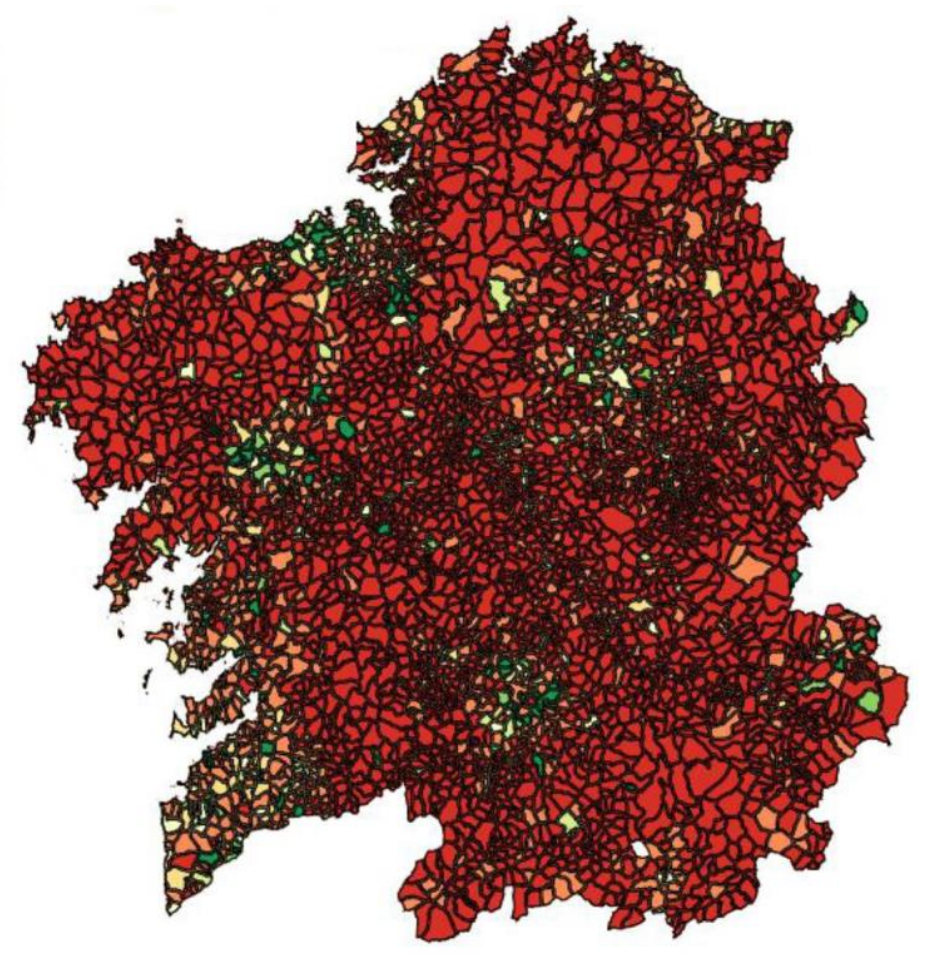

Galicia

\% var. pob. 2019-2033

Más de 20

$(100,20]$

$(5,10]$

$(0,5]$

$(-5,0]$

$(-20,-5]$

Menos de -20

Figura 6. Variación porcentual estimada de la población parroquias gallegas, 2019-2033. Fuente: elaboración propia. 


\section{Conclusiones}

Asistimos a una era de fuerte concentración de la población alrededor de las grandes ciudades que está dejando despobladas las zonas rurales. Existe una amplia literatura que explora, desde distintas perspectivas, las causas de estos procesos de concentración. No obstante, frente a ello, los estudios de lo que ocurre en áreas que tienden a perder población, zonas rurales y pequeños núcleos urbanos, son más limitados debido, en muchas ocasiones, a la ausencia de información con suficiente nivel de desagregación espacial. Por ejemplo, en el caso de España, el Instituto Nacional de Estadística ofrece proyecciones de población por provincias, sin ser posible anticipar las dinámicas intraprovinciales. No disponemos, por tanto, de proyecciones de población, envejecimiento, tasa de natalidad o densidad de población para los entornos rurales y zonas periféricas protagonistas habituales del declive demográfico.

En este trabajo hemos propuesto un sencillo procedimiento para desagregar las proyecciones del INE a una escala mucho más detallada que la provincia, que nos permite tener información de las tendencias previsibles en zonas rurales o en pequeños núcleos poblacionales totalmente consistentes con las proyecciones oficiales a escala provincial. Gracias a ello, puede convertirse en una herramienta muy valiosa para ayudar a la toma de decisiones y al diseño de agendas demográficas que tengan en cuenta la heterogeneidad de comportamientos intrarregionales que se observan en la realidad.

A modo de ilustración se ha aplicado dicho procedimiento a Asturias y a Galicia, estimando proyecciones de la densidad de población por parroquias para el período 2019-2033. Las parroquias, como ya se ha indicado con anterioridad, son entidades de ámbito territorial inferiores al municipio. Entre las dos regiones suman 4.643 parroquias, más de diez veces superior al número de municipios, lo que nos permite tener una visión extraordinariamente detallada de las perspectivas poblacionales en estas dos regiones.

Los resultados de nuestra aplicación son coherentes con las conclusiones de algunos estudios previos que han trabajado con áreas ampliamente desagregadas. Observamos una tendencia a la concentración alrededor de las principales ciudades del área estudiada. Dentro de las áreas rurales, constatamos un comportamiento similar, con un abandono progresivo de la aldea, o el asentamiento rural disperso, en favor de una reconcentración alrededor de los núcleos de población principales de cada área. Todo ello conduce a un cambio de modelo de asentamiento poblacional. Cada vez se ocupa menos territorio, y el espacio despoblado se acerca a los núcleos urbanos.

Nuestros resultados contradicen la creencia de que en el contexto de la sociedad de la información y la comunicación asistiremos a un proceso de migración desde los principales núcleos urbanos a los entornos rurales y, por tanto, a la reconquista del "campo". Al menos hasta la fecha, y en las proyecciones hechas hasta el año 2033, no se aprecia que ese comportamiento se produzca de modo relevante. Lo que observamos, por el contrario, es una tendencia a la concentración cerca de las ciudades o delas áreas más pobladas. Las políticas de ordenación del territorio y conservación medioambiental deberían tener en cuenta esta tendencia, planificando la reforestación de montes y la creación de espacios naturales más amplios ante un modelo de asentamiento de la población que tiende a ser mucho más concentrado.

\section{Apéndice 1. Mapas de las provincias gallegas de variaciones porcentuales estimadas, 2019-2033}

Como ya hemos señalado anteriomente, en las Figuras 7 a 10 presentamos la variación porcentual estimada de la población de las parroquias de las provincias de A Coruña, Lugo, Ourense y Pontevedra en el período 2019-2033. 


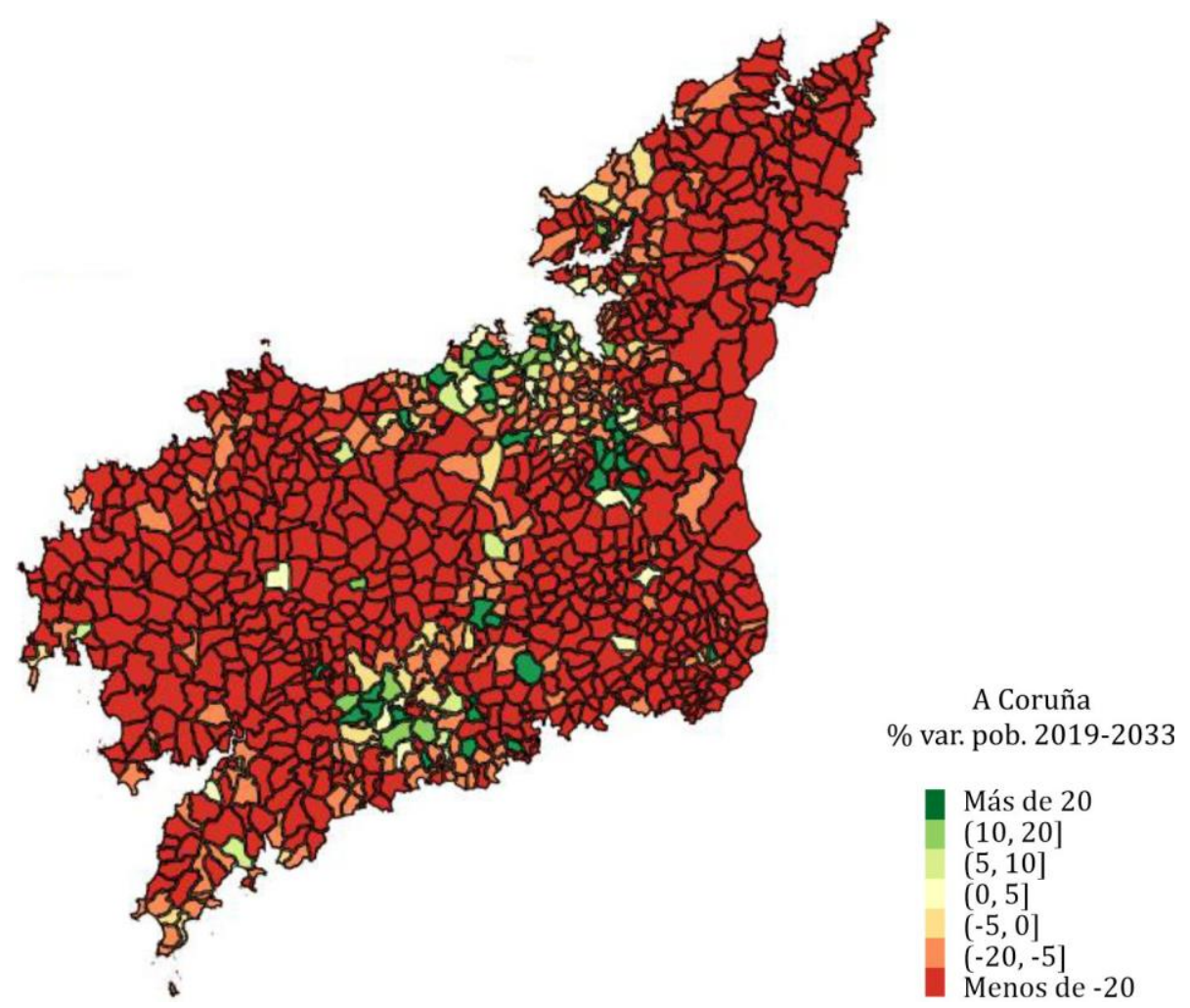

Figura 7. Variación porcentual estimada de la población de las parroquias de A Coruña, 2019-2033. Fuente: elaboración propia.

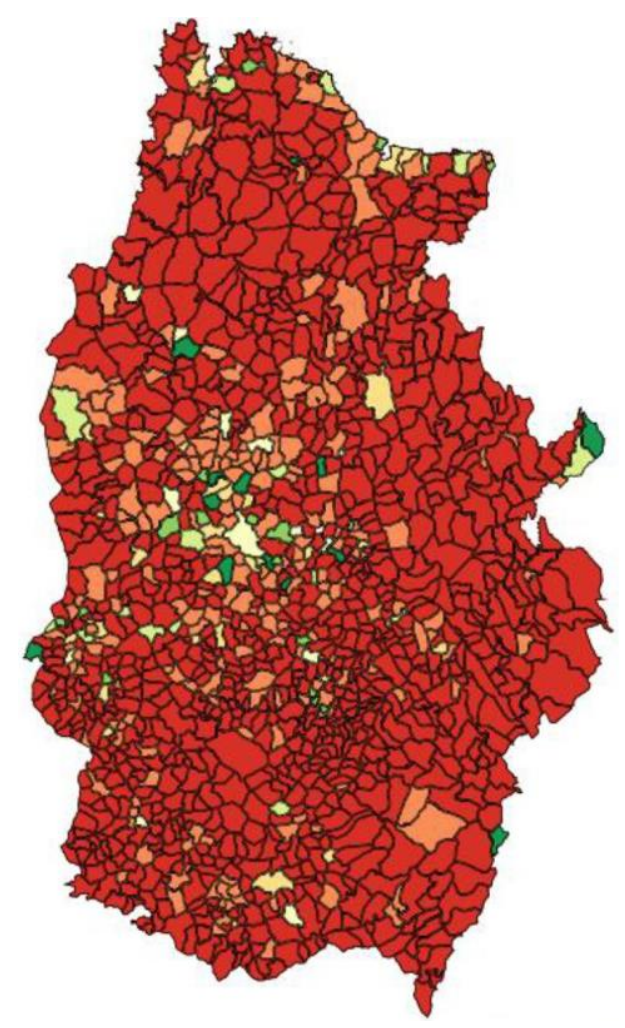

\section{Lugo}

\% var. pob. 2019-2033

Figura 8. Variación porcentual estimada de la población de las parroquias de Lugo, 2019-2033. Fuente: elaboración propia. 


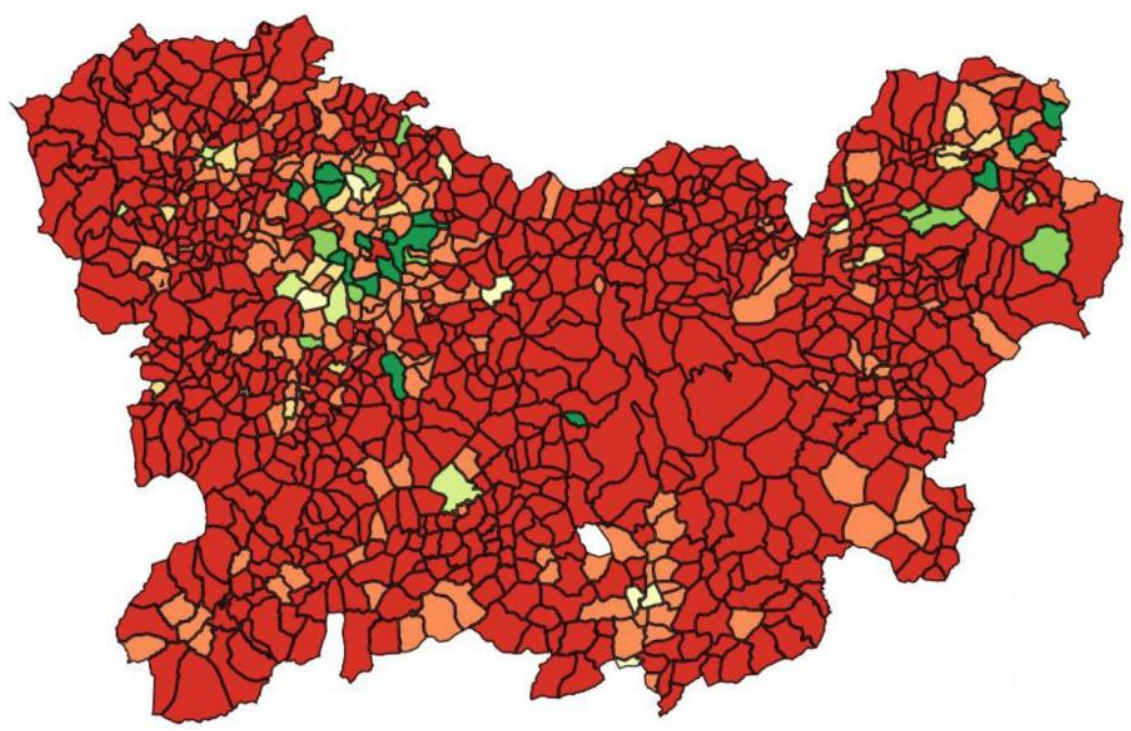

Ourense

$\%$ var. pob. 2019-2033

Más de 20
$(10,20]$
$(5,10]$
$(0,5]$
$(-5,0]$
$(-20,-5]$
Menos de -20

Figura 8. Variación porcentual estimada de la población de las parroquias de Lugo, 2019-2033. Fuente: elaboración propia.

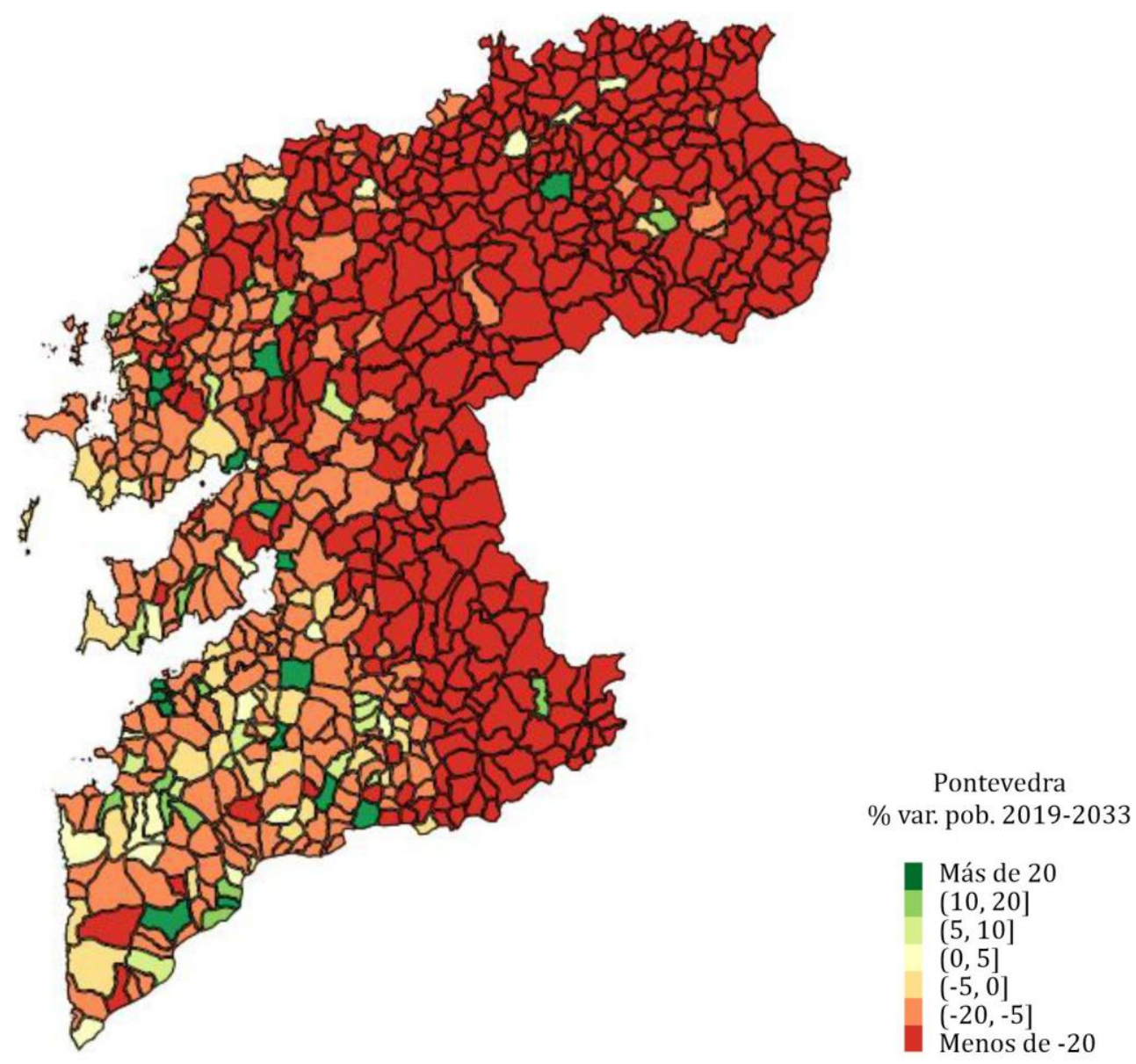

Figura 8. Variación porcentual estimada de la población de las parroquias de Lugo, 2019-2033. Fuente: elaboración propia. 


\section{Apéndice 2. Variación porcentual de la población parroquias asturianas y gallegas, 2005-2019}

Por último y también como hemos señalado con anterioridad, en las Figuras 11 y 12 recogemos la variación porcentual estimada de la población de las parroquias asturianas y gallegas en el intervalo 2005-2019.

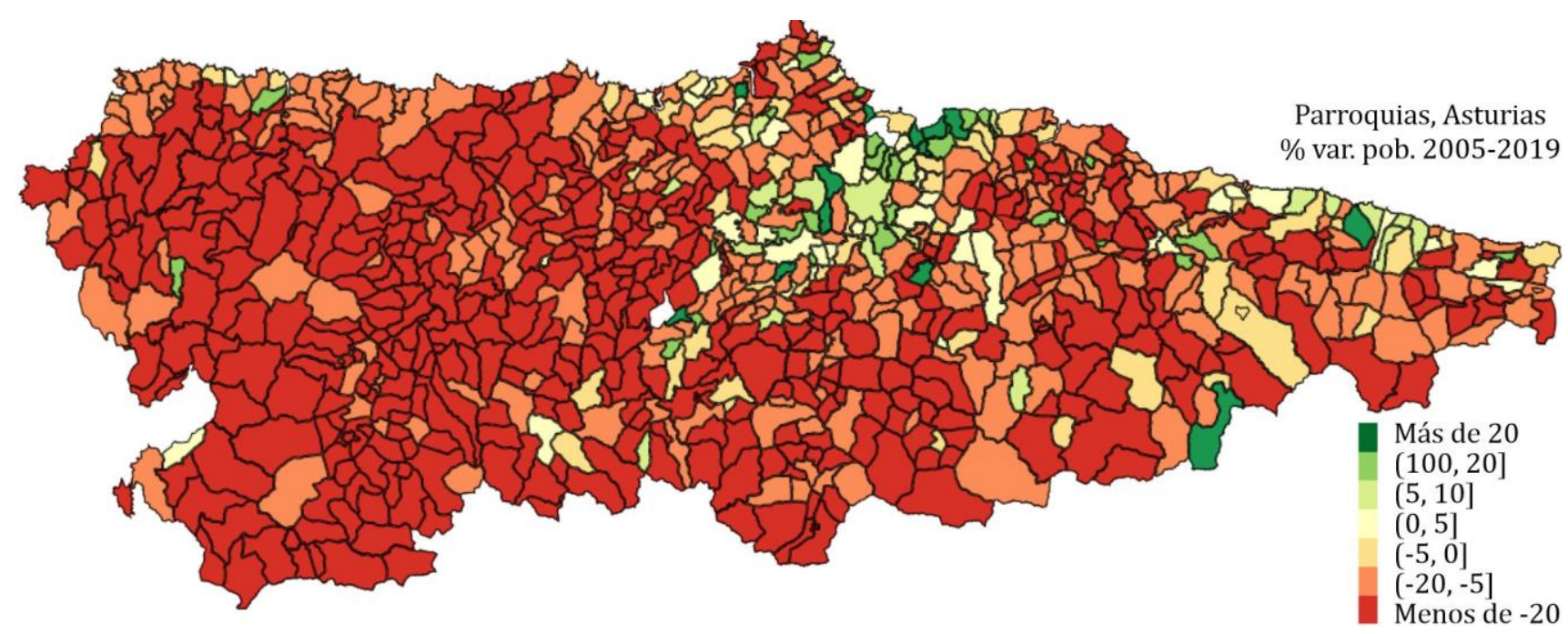

Figura 11. Variación porcentual estimada de la población de las parroquias de Asturias, 2019-2033. Fuente: elaboración propia.

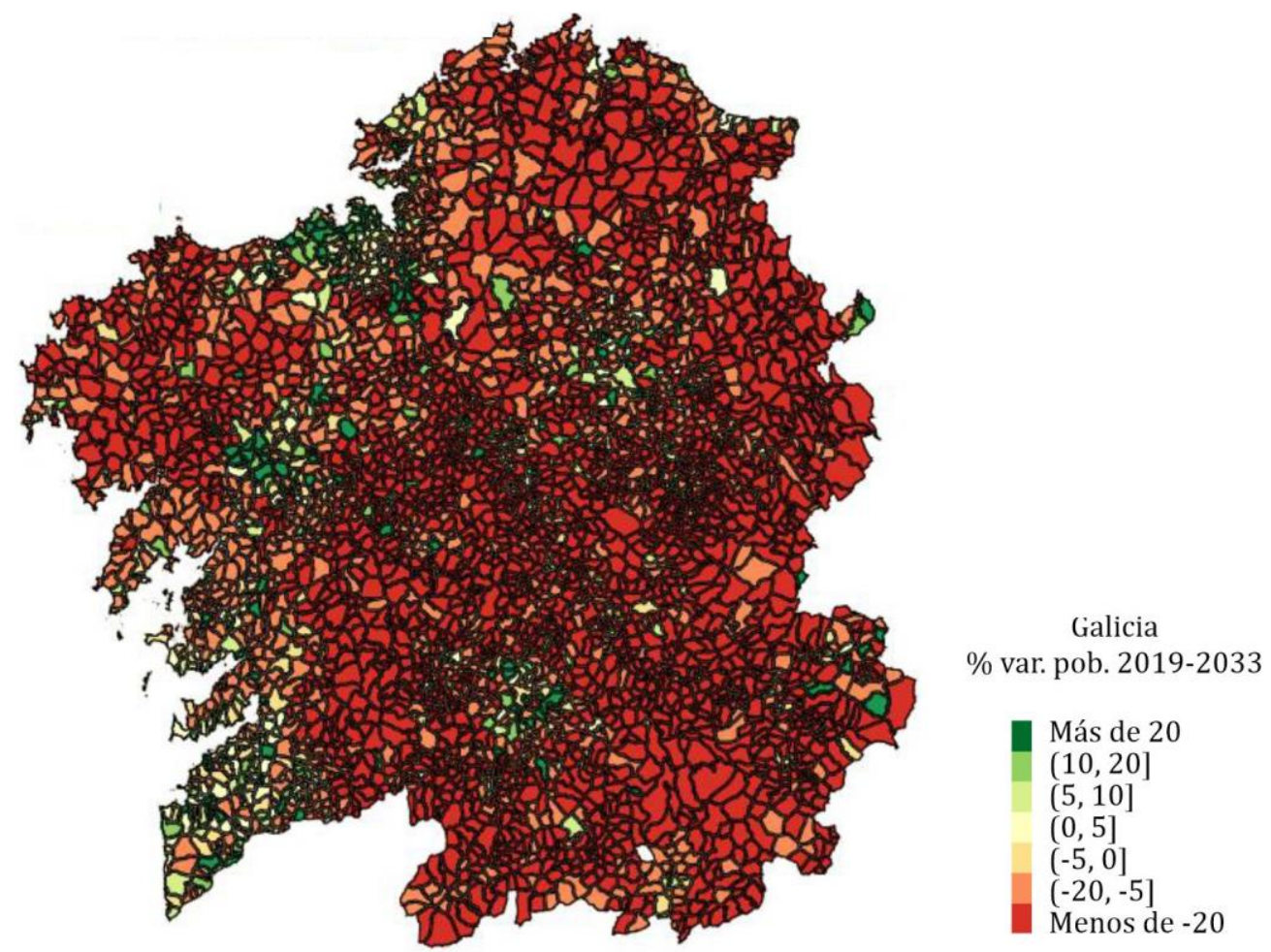

Figura 12. Variación porcentual estimada de la población de las parroquias de Galicia, 2019-2033. Fuente: elaboración propia. 


\section{Bibliografía}

Alonso, M.L., Fernández Vázquez, E., y Rubiera, F. (2015). A methodological note for local demographic projections: A shift-share analysis to disaggregate official aggregated estimations. Rect@: Revista Electrónica de Comunicaciones y Trabajos de ASEPUMA, 16(1), 43-50.

Recuperado de: https://dialnet.unirioja.es/servlet/articulo?codigo=5601444

European Commission. (2020). Global Human Settlement Layer (GHSL). Brussels, Belgium: European Commission. Research Group, Joint Research Center.

Recuperado de: https://ec.europa.eu/jrc/en/global-human-settlement-layer

Dunn, E. S. (1960). A statistical and analytical technique for regional analysis. Papers of the Regional Science Association, 6, 97-112. DOI: https://doi.org/10.1111/i.1435-5597.1960.tb01705.x

Glaeser, E.L., Kourtit, K., y Nijkamp, P. (2020). Urban empires: Cities as global rulers in the new urban world. New York, NY: Routledge.

Glaeser, E.L., Ponzetto, G. A. M., y Tobio, K. (2014). Cities, skills and regional change. Regional Studies, 48(1), 7 43. DOI: https://doi.org/10.1080/00343404.2012.674637

Gutiérrez, D., Rubiera, F., y Viñuela, A. (2017). Heterogeneity in the determinants of the population growth at the local level. Analysis of the Spanish case with GWR approach. International Regional Science Review, 40 (3), 211-240. DOI: https://doi.org/10.1177/0160017615589009

King, G., Rosen, O., y Tanner, M.A. (2004). Ecological inference: New methodological strategies. Cambridge, UK: Cambridge University Press. DOI: http://doi.org/10.1017/CB09780511510595

Mayor, M., López, A. J., y Pérez, R. (2007). Forecasting regional employment with shift-share. Regional Studies, 41 (4), 543-551. DOI: https://doi.org/10.1080/00343400601120205

Mazzuco, S., y Keilman, N. (Eds.). (1990). Developments in demographic forecasting.The Springer Series on Demographic Methods and Population Analysis, 49. Cham, Switzerland: Springer. DOI: https://doi.org/10.1007/978-3-030-42472-5

Rubiera, F., Fernández, E., Gutiérrez, D., y Viñuela, A. (2014). Análisis de la situación y perspectivas demográficas en el Principado de Asturias y revisión de las políticas aplicadas en Europa para el diseño de una agenda demográfica. Primer informe del diagnóstico de la situación y perspectivas demográfricas del Principado de Asturias. Oviedo: Laboratorio de Análisis Económico y Regional -REGIOlab. 